\title{
MIMO Optical Wireless Channels Using Halftoning
}

\author{
Mohamed D. A. Mohamed, Awad Dabbo and Steve Hranilovic \\ Dept. Electrical \& Computer Engineering, McMaster University \\ Hamilton, Ontario, Canada \\ Email: \{mdamohamed, dabboab\}@grads.ece.mcmaster.ca, hranilovic@mcmaster.ca
}

\begin{abstract}
Two-dimensional (2D) optical intensity channels exist in a variety of applications including holographic storage, page-oriented memories, optical interconnects, 2D barcodes, as well as MIMO wireless optical links. This paper considers the capacity of such channels when the transmitted signal is binarylevel. Strict spatial alignment between transmitter and receiver is not required nor is independence among the spatial channels. Spatial discrete multitone modulation is combined with digital image halftoning to produce a binary-level transmit image. Unlike earlier work, this paper considers imagers with pixels of fixed size and quantifies the tradeoff between frame rate, array size and capacity per frame. An experimental prototype pixelated wireless optical channel is constructed, and the channel parameters are measured. With a measured channel model, rates on the order of $450 \mathrm{Mbps}$ are predicted for a $1 \mathrm{~m}$ link using 0.5 megapixel arrays at a frame rate of $7 \mathrm{kfps}$.
\end{abstract}

\section{INTRODUCTION}

Two-dimensional (2D) optical intensity channels transmit information by modulating the instantaneous intensity of an array of optical sources. That is, the transmitted signal is an optical intensity image, and hence is constrained to be non-negative. Applications that use such channels are holographic data storage [1,2], page-oriented optical recording [3], 2D barcodes [4], and multiple-input/multiple-output (MIMO) wireless optical communications [5,6]. A key implementation issue with these applications is the requirement of strict spatial alignment between the transmitter and the receiver to avoid inter-channel interference $[1,7]$. Such systems are called pixel matched, i.e. each receive pixel images a single transmit pixel.

Spatial discrete multitone (SDMT) modulation has been introduced for 2D intensity channels in [8]. It provides high spectral efficiencies without the need for strict spatial alignment between the transmitter and the receiver. In [8], the transmitter array is assumed to be able to generate multiple (e.g., 256) intensity levels. Additionally, the non-negativity constraint of the generated image is not considered explicitly, rather, amplitudes are clipped to ensure that the constraints are met. We have proposed halftoned-SDMT modulation in [9] as an extension to SDMT. It considers transmitter arrays, i.e., spatial light modulators (SLMs), in which each pixel is able to output a binary-level intensity, i.e., on or off. Such transmitters are far simpler than the multiple-level ones and can be operated at very high frame rates. The non-negativity constraint is treated explicitly through the design of modified halftoning algorithms. Thus, excess degrees of freedom in spatial frequency domain are exploited to provide binary-level output images which satisfy all amplitude constraints while maintaining the relaxation of the strict spatial alignment.

The size of the imager receiver was assumed constant in [9], and hence increasing the number of receiving pixels was achieved by decreasing the pixel size. Moreover, the effect of the frame rate on channel capacity was not studied. In this work, a more practical assumption of having a fixed pixel size is considered. This allows for the signal-to-noise ratio (SNR) to be independent of the number of pixels. An experimental prototype of the channel is presented along with measurements of the channel parameters. The effect of the number of framesper-second (fps) and the array size, i.e. number of pixels, on the SNR and the channel capacity is studied. As shown in Sec. IV-B, maximum capacity, in Mbps, is achieved when the degradation in SNR with increasing frame rate is balanced by a larger number of fps.

\section{Channel Model}

For the MIMO optical channels considered here, the transmitter is an SLM consisting of an array of pixels, while the receiver is a $2 \mathrm{D}$ array of photodetector elements. The receiver samples the spatial distribution of the optical intensity wavefront incident on its surface. Examples of such receivers are arrays of photodiodes and CMOS imagers. Two amplitude constraints are imposed on the transmit image: (i) a non-negativity constraint since the transmitted signal is an optical intensity, and (ii) the average image amplitude is upper bounded by the average optical power.

Imaging optics are employed such that the receive image is an orthographic projection of the transmit image. Imaging optics and transmit/receive pixel shapes can be modelled as a lowpass linear space-invariant (LSI) system characterized by a point-spread function (PSF) [10]. This channel model is a generalization of a previous model used in holographic data storage [2], 2D optical recording [3] and wireless optical communications [11], where the channel was modelled as a 2D finite-extent LSI system that represents the optical blur. Only indoor channels are considered in this work, and hence atmospheric turbulence and fading effects are not considered. Gaussian distributed noise corrupts each receiver pixel as in previous optical wireless studies $[8,12]$.

SDMT is a modulation scheme developed for spatially frequency selective channels [8]. Data are modulated in spatial frequency and a transmit image is formed by taking the inverse 2D discrete Fourier transform (DFT). By appending a cyclic extension [8] around the image, whose size is at least half the channel memory, equalization is simplified to a complex 
multiplication per spatial frequency bin. In this case, the channel model can be written in discrete spatial frequency domain $\left(k_{1}, k_{2}\right)$ as

$$
R\left(k_{1}, k_{2}\right)=X\left(k_{1}, k_{2}\right) H\left(k_{1}, k_{2}\right)+Z\left(k_{1}, k_{2}\right)
$$

where $R, X, H$, and $Z$ are the DFTs of the receive image, transmit image, PSF, and channel additive Gaussian noise respectively. Despite the fact that the PSF models the correlation between the pixels in the spatial intensity domain, the MIMO channel can be considered as a number of independent parallel Gaussian channels in spatial frequency domain as in (1). Therefore the aggregate capacity, in the absence of amplitude constraints, is maximized by a water pouring algorithm over the spatial frequency channels [8]. However, due to the nonnegativity amplitude constraint of the spatial intensity domain, pouring power to the spatial frequency channels is not straight forward, and does not necessarily maximize the capacity [8]. Moreover, an SLM capable of outputting a continuous high dynamic range of intensities is required.

\section{BINARY-LEVEL SignALING}

Restricting the amplitudes of the SLM to be binary-level not only reduces complexity, but ensures that the channel nonnegativity constraint can be easily met since only dark/bright pixels are transmitted over the channel. Examples of binarylevel SLMs include digital micro-mirror devices (DMD) and arrays of vertical-cavity surface-emitting lasers (VCSELs). Commercial DMD can operate at switching speeds of nearly $10 \mathrm{kHz}$ with array sizes of $2048 \times 1152$ mirrors [13].

Since the channel is spatially low pass, transmission of data in high spatial frequency modes is not possible since these modes are effectively filtered by the channel. The highfrequency modes are termed the out-of-band region, while the low-frequency modes which carry independent data are termed the in-band region. Our technique exploits these out-of-band spatial modes to satisfy the channel non-negativity constraint by producing binary-level output images while achieving high communication rates that approach the rates of unconstrained continuous SLM transmission.

\section{A. Digital Image Halftoning}

Digital image halftoning is the process of converting a continuous-tone image to a binary-level one which is perceptually similar to the original image [14]. This conversion is achieved via the careful addition of quantization noise to the continuous-tone image in spatial frequency bins which are beyond the perceptual range. The most popular halftoning algorithm is error diffusion halftoning, first proposed empirically in [15]. Later, it was shown that the error diffusion algorithm is the extension of $\Delta \Sigma$ modulation to two dimensions [16]. The error diffusion system used in this work is surrounded by a dashed box in Fig. 1. Rigorous design techniques based on filter theory have been also been proposed for $j\left(n_{1}, n_{2}\right)$ [17].

For 2D optical intensity communication channels, halftoning is used to produce a binary-level transmit image in which the quantization noise is shaped to the out-of-band frequency

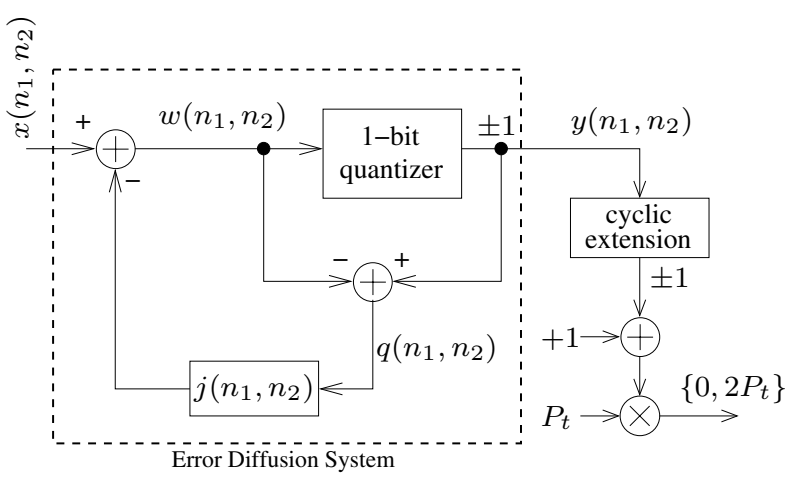

Fig. 1. Block Diagram of halftoned SDMT system.

bins that are heavily attenuated by the channel PSF. This high frequency quantization noise will be filtered out by the channel and the received image will be a continuous-tone image which carries the transmitted data along with some residual quantization noise. The feedback filter $j\left(n_{1}, n_{2}\right)$ in Fig. 1 is designed so as to maximize the channel capacity.

In Fig. 1, let $q$ be the quantizer error, and $\tilde{q}=y-x$ be the closed loop quantization noise, where $x$ and $y$ are the input continuous-tone image and the output halftoned image respectively. A conventional assumption is to linearize the quantizer by assuming that the quantizer error $q$ is white with variance $\sigma_{q}^{2}$ and signal independent [18], [19, Chapter 14]. Once linearized, the following power spectral density (PSD) of the quantization noise $\tilde{q}$ can be easily deduced

$$
\Phi_{\tilde{q}}\left(k_{1}, k_{2}\right)=N_{1} N_{2} \sigma_{q}^{2}\left|1-J\left(k_{1}, k_{2}\right)\right|^{2},
$$

where $N_{1} \times N_{2}$ is the image size before adding the cyclic extension, and $J\left(k_{1}, k_{2}\right)$ is the DFT of the feedback filter. The noise power shaping function, $\left|1-J\left(k_{1}, k_{2}\right)\right|^{2}$, is designed to shape the quantizer error power to the out-of-band region [9]. In order for this model to be consistent and for the modulator to remain stable, the average electrical power of the input SDMT signal, $\sigma_{x}^{2}$, must be limited as justified in [9].

\section{B. Halftoned Spatial Discrete Multitone Modulation}

Figure 1 presents the binary-level SDMT communication system based on halftoning. An SDMT symbol is formed and input to the halftoning algorithm. A cyclic extension is added to the halftoned image and the frame is biased to satisfy the non-negativity constraint. Finally the transmitted image is scaled by $P_{t}$ to have output levels $\left\{0,2 P_{t}\right\}$, and hence the average transmit optical power is equal to $P_{t}$.

The received image is equalized by dividing it by the channel transfer function and $P_{t}$,

$$
\begin{aligned}
\frac{R\left(k_{1}, k_{2}\right)}{P_{t} H\left(k_{1}, k_{2}\right)}= & {\left[X\left(k_{1}, k_{2}\right)+N_{1} N_{2} \delta\left(k_{1}, k_{2}\right)\right] } \\
& +\tilde{Q}\left(k_{1}, k_{2}\right)+\tilde{Z}\left(k_{1}, k_{2}\right)
\end{aligned}
$$

where $\tilde{Z}\left(k_{1}, k_{2}\right)=Z\left(k_{1}, k_{2}\right) /\left(P_{t} H\left(k_{1}, k_{2}\right)\right)$ is the effective channel Gaussian noise. That is, the equalized image is equal to a DC-biased version of the transmit image contaminated 
by two noise components: quantization noise, $\tilde{Q}\left(k_{1}, k_{2}\right)$, and channel noise, $\tilde{Z}\left(k_{1}, k_{2}\right)$, with PSDs $\Phi_{\tilde{q}}\left(k_{1}, k_{2}\right)$ and $\Phi_{\tilde{z}}\left(k_{1}, k_{2}\right)$ respectively.

Due to the equalization in (3), the image can be considered as an array of independent parallel complex Gaussian channels in frequency domain. Notice, however, that half the complex channels are dependent on the other half due to conjugate symmetry [9]. Therefore the capacity, $C$, is maximized by using the water pouring algorithm to define the electrical power, $\Phi_{x}\left(k_{1}, k_{2}\right)$, allocated to bin $\left(k_{1}, k_{2}\right)$ such that the total input electrical power constraint is met [9]. The non-negativity constraint is no longer an issue during the water pouring power allocation, as was the case in [8], since the output image is a binary one. No power is poured to the DC-bin since a constant bias of $P_{t}$ is added to each frame.

\section{EXAmple: Pixelated Wireless Optical Channel}

In this section, an experimental prototype of a pixelated wireless optical channel has been constructed to measure the salient channel parameters to enable the creation of accurate channel models. For purposes of design, it is assumed in our simulations that the channel noise is white, Gaussian, and signal independent with variance $\sigma_{z}^{2}$. Therefore,

$$
\Phi_{\tilde{z}}\left(k_{1}, k_{2}\right)=\frac{\sigma_{z}^{2} N_{1} N_{2}}{\left|P_{t} H_{0} H\left(k_{1}, k_{2}\right)\right|^{2}}=\frac{N_{1} N_{2}}{\left|\rho H\left(k_{1}, k_{2}\right)\right|^{2}},
$$

where $H_{0}$ is the DC-gain, $H\left(k_{1}, k_{2}\right)$ is unity DC-gain (assumed to be Gaussian-shaped with normalized widths $w_{h_{1}}$ and $\left.w_{h_{2}}\right)$, and

$$
\rho=P_{t} H_{0} / \sigma_{z} .
$$

The receiver is assumed to be $N \times N$ pixels, i.e. $N_{1}=N_{2}=$ $N$, while the transmitter is assumed to be $(N+M) \times(N+M)$, where $M$ is the width of the cyclic extension. The value of $M$ is at least half the channel memory, i.e. $M$ is a function of the PSF extent. Using (2) and (4), the capacity is equal to

$$
\begin{aligned}
C= & \frac{1}{2} \sum_{k_{1}=0}^{N_{1}-1} \sum_{k_{2}=0}^{N_{2}-1} \log \left(1+\frac{\Phi_{x}\left(k_{1}, k_{2}\right)}{N_{1} N_{2}} /\right. \\
& {\left.\left[\sigma_{q}^{2}\left|1-J\left(k_{1}, k_{2}\right)\right|^{2}+\left|\rho H\left(k_{1}, k_{2}\right)\right|^{-2}\right]\right) . }
\end{aligned}
$$

If $\rho \gg 1 / \sigma_{q}$, the quantization noise dominates and the system is termed quantization noise-limited. On the other hand, if $\rho \ll 1 / \sigma_{q}$, the channel noise dominates and the system is then termed optical power-limited.

Spatial synchronization is achieved through appropriate scaling of the imaging optics [8]. Therefore, the transmitter and receiver have the same spatial Nyquist region. As designed in [9], the feedback filter is taken as

$$
j\left(n_{1}, n_{2}\right)=\left[\begin{array}{cc}
0 & 0.9 \\
0.9 & -0.8
\end{array}\right],
$$

which is amenable to implementation and has unity DC-gain [9]. As $\sigma_{x}^{2}$ increases, so too does the likelihood of quantizer saturation and system instability. In the experimental results that follow, $\sigma_{x}^{2}=0.2$ is used. This value of $\sigma_{x}^{2}$ satisfies the upper bound given in [9] that is necessary for the modulator stability. At this value of $\sigma_{x}^{2}$, the correlation between the signal and the quantization noise is about $2.5 \%$. The higher $\sigma_{x}^{2}$, the higher the correlation, which violates the aforementioned assumption of signal-independent quantization noise.

An example of a continuous-tone image is shown in Fig. 2 for a frame rate of $7142 \mathrm{fps}$, along with the corresponding halftoned image and their spectra. Data are loaded in the low frequency bins of the continuous-tone image as implied by the water pouring allocation. Comparing the spectra in Figs. 2(c) and 2(d), it is clear that a great majority of the quantization noise power is shaped to the out-of-band region, which is indicated by the four high power corners of the halftoned image spectrum. In this case, the system is optical power-limited and the shape of the in-band power allocation is dominated by the PSF.

A similar example for a frame rate of $400 \mathrm{fps}$ is shown in Fig. 3. The channel noise at this frame rate is far lower than that at the higher frame rate of Fig. 2, i.e. higher value of $\rho$. As a result, the shape of the in-band power allocation is dominated by the quantization noise shaping function $\left|1-J\left(k_{1}, k_{2}\right)\right|^{2}$, and the system is quantization noise-limited. Qualitatively, data cannot be fully recovered from the low frequency region of the spectrum of the halftoned image because of the in-band quantization noise.

\section{A. Experimental Setup}

The transmitter is based on a commercial DLP projector [20, LT30] which projects its image onto a flat wall. The circuitry of the projector is replaced with a controller board for the DLP which allows freedom to transmit any sequence of stored images [13]. This configuration allows for frame rates of up to $10 \mathrm{kfps}$ and a maximum resolution of $1024 \times 768$ pixels. The illumination source of the projector has been replaced with a metal halide machine vision illuminator. The illuminator was coupled to the projector optics using a $1 / 2$ " fiber optic lightguide as shown in Fig. 4.

The receiver is a high speed mega-pixel CMOS digital camera [21, MC1310] which captures the image projected on the wall. The camera is connected to a frame grabber [22, Odyssey XCL] using the full camera Link configuration which is capable of transferring data at a maximum rate of 660 Mbytes/sec. Digital recording software [23, Streampix] is used to capture and store the received frames. Temporal synchronization is achieved by using a signal generator card [24, NI6601] that generates trigger pulses that drive both the transmitter and receiver. When a trigger pulse is received by the projector, an image is projected onto the wall. After few micro-seconds delay, the camera starts integrating the spatial intensity image. The integration time of the camera is controlled by a programmable shutter, with exposure time less than the illumination period of the frame.

The separation distance between the camera, and the projected image is set to $1.1 \mathrm{~m}$. The camera was positioned to ensure that its optical axis aligns with that of the image in order to minimize the projective distortion. The focal length 


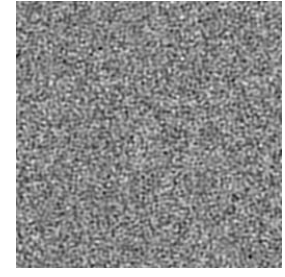

(a) continuous-tone image

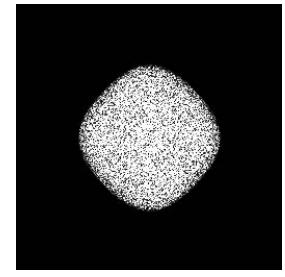

(c) continuous-tone image spectrum

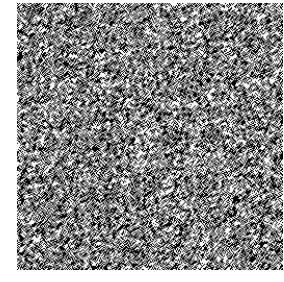

(b) halftoned binary-level image

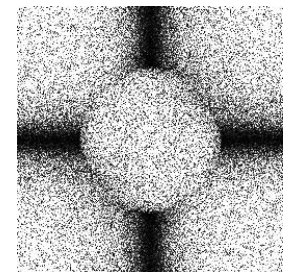

(d) halftoned binary-level image spectrum
Fig. 2. A continuous-tone image (a), corresponding halftoned image (b), and their spectra (c, d) for $7142 \mathrm{fps}, N=256, \sigma_{x}^{2}=0.2, w_{h_{1}}=49.57$, $w_{h_{2}}=48.29$, and $\rho=2.13$. This is an optical power-limited system.

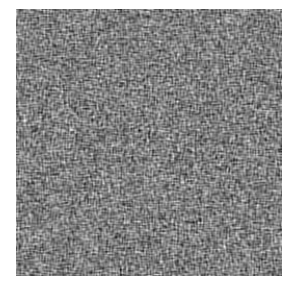

(a) continuous-tone image

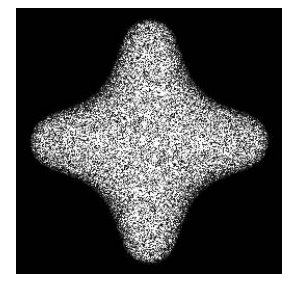

(c) continuous-tone image spectrum

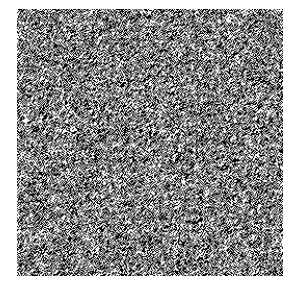

(b) halftoned binary-level image

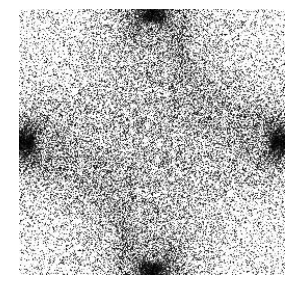

(d) halftoned binary-level image spectrum
Fig. 3. A continuous-tone image (a), corresponding halftoned image (b), and their spectra (c, d) for $400 \mathrm{fps}, N=256, \sigma_{x}^{2}=0.2, w_{h_{1}}=49.57$, $w_{h_{2}}=48.29$, and $\rho=20.83$. This is a quantization noise-limited system.

of the projector was adjusted to get unity magnification (i.e. image size in pixels is the same at both transmitter and the receiver). To mitigate hardware limitations and generate consistent measurements, the operating frame rate was fixed to $100 \mathrm{fps}$, and the programmable shutter is used to adjust the exposure time such that the effective frame rate is given by the inverse of the exposure time. As the exposure time changes, the sensor gain is manually adjusted such that the histogram of the received image occupies the full dynamic range (0 to 255) of the sensor.

Channel noise measurements were performed by transmitting and receiving sequences of dark and illuminated frames. The channel noise consists of fixed pattern noise, due to vari-

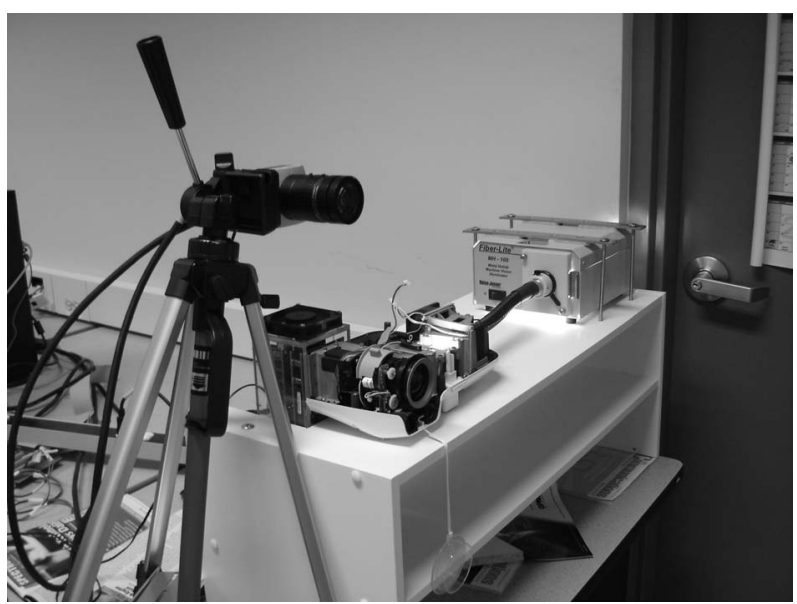

Fig. 4. MIMO link prototype setup

ations over the sensor array, and signal independent noise. For each frame rate, the fixed pattern noise is estimated by sending 1000 dark frames and averaging over the received frames. The signal independent noise is estimated by averaging over 1000 dark frames and 1000 illuminated frames. By assuming that the noises of neighboring pixels are independent, the complex noise in discrete frequency domain is white.

The channel PSF is measured by sending a narrow spatial pulse $(2 \times 2$ pixels) at different positions in space (top left, top right, center, bottom left, and bottom right). For each pulse position, 1000 frames where captured and the average computed. The measured response is fitted to a 2D Gaussian shape whose widths are $w_{h_{1}}=49.57$ and $w_{h_{2}}=48.29$.

\section{B. Discussion}

The capacity of the halftoned SDMT system, or any finitelevel SDMT system, is upper bounded by that of a continuous SDMT transmitter with the same $\sigma_{x}^{2}$ neglecting the nonnegativity amplitude constraints. This upper bound is given by (6) by setting $\sigma_{q}^{2}=0$ and also serves as an upper bound to previous results in [8]. The capacity of the halftoned SDMT system, measured in kbits/frame and Mbps, is compared to this upper bound in Figs. 5 and 6 respectively.

At a given frame size, the capacity per frame decreases with the frame rate in Fig. 5. This is because the channel noise variance increases linearly with the frame rate. Therefore, as in (5), $\rho$ varies inversely as the square root of the frame rate. For example, as in Figs. 2 and 3, the measured values of $\rho$ are 2.13 and 20.83 at frame rates 7142 and 400 fps respectively. Hence the system tends to be optical power-limited as the frame rate increases, and the capacity of the binary-level transmitter approaches the upper bound as shown in Figs. 5 and 6.

On the other hand, the capacity in Mbps increases with frame rate, as in Fig. 6, at low and moderate frame rates. This is because, as the frame rate increases, the information transmitted per second increases until the degradation in $\rho$ dominates the effect of the faster frame rates. This fact is evident in Fig. 6, where a maximum capacity is achieved at frame rate of $7142 \mathrm{fps}$. 


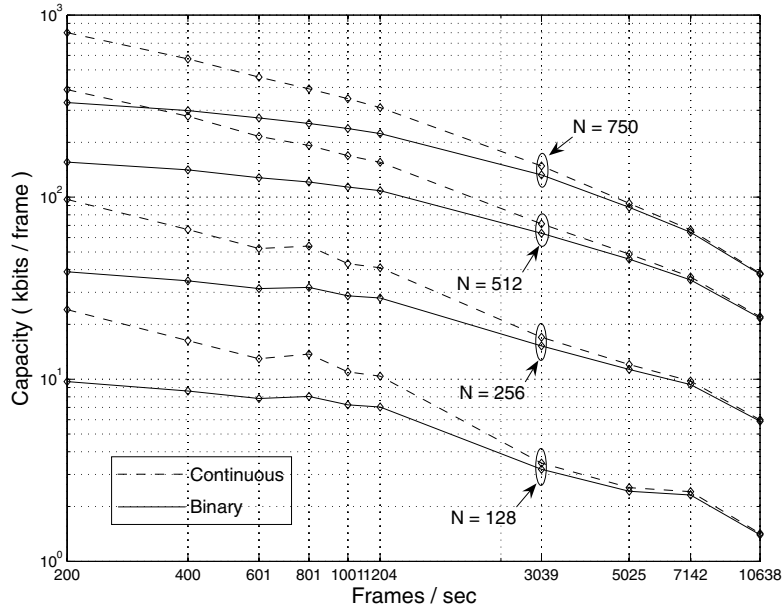

Fig. 5. Capacity of the pixelated channel, in kbits/frame, versus frame rate with $\sigma_{x}^{2}=0.2$. The linearized analytical model in Sec. III-A is compared to a continuous SDMT transmitter without non-negativity constraint.

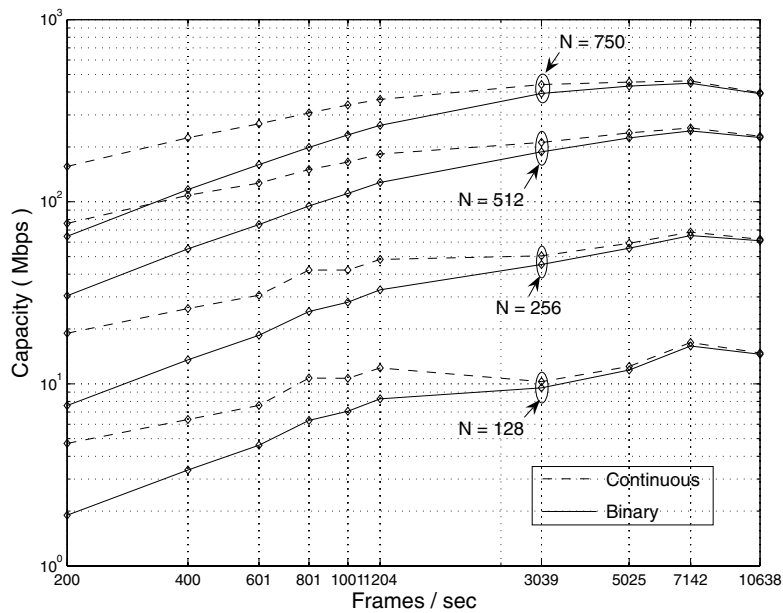

Fig. 6. Capacity of the pixelated channel, in Mbps, versus frame rate with $\sigma_{x}^{2}=0.2$. The linearized analytical model in Sec. III-A is compared to a continuous SDMT transmitter without non-negativity constraint.

It is worth noting that, at a given frame rate, the capacity increases proportional to the number of pixels $N^{2}$. This is due to the fact that the sizes of both the in-band and out-ofband regions increase with the same proportion. That is, if $N$ is doubled, the number of complex frequency channels is multiplied by four, and so approximately is the capacity.

\section{CONCLUSION}

In this work, we have considered the design of point-topoint 2D optical intensity channels in which binary-level optical intensity transmitters are used. An experimental pixelated wireless optical channel is constructed to develop an accurate channel model. It is shown that with a simple feedback filter, the capacity of the channel using a binary-level transmitter approaches that when using an idealized continuous transmitter. Additionally, we demonstrate that the frame rate of such systems must be carefully chosen to maximize the capacity in Mbps. The goal of this work is to demonstrate that the fundamental limits of pixelated channels with binarylevel transmission are significant and merit continued study. Our current directions include continued development of the prototype and coding techniques.

\section{REFERENCES}

[1] R. M. Shelby, J. A. Hoffnagle, G. W. Burr, C. M. Jefferson, M.-P. Bernal, H. Coufal, R. K. Grygier, H. Gunther, R. M. Macfarlane, and G. T. Sincerbox, "Pixel-matched holographic data storage with megabit pages," Optics Letters, vol. 22, no. 19, pp. 1509 - 1511, Oct. 1997.

[2] S. Nabavi and B. V. K. V. Kumar, "Detection methods for holographic data storage," in Proceedings of the IEEE Optical Data Storage Topical Meeting, Montreal, QC, Canada, 23-26 Apr. 2006, pp. 156 - 158.

[3] P. H. Siegel, "Information-theoretic limits of two-dimensional optical recording channels," in Proceedings of the IEEE Optical Data Storage Topical Meeting, Montreal, QC, Canada, 23-26 Apr. 2006, pp. 165- 167.

[4] R. Villn, S. Voloshynovskiy, O. Koval, and T. Pun, "Multilevel 2-D bar codes: Toward high-capacity storage modules for multimedia security and management," IEEE Trans. Inf. Forens. Security, vol. 1, no. 4, pp. 405-420, Dec. 2006.

[5] D. C. O'Brien, S. Quasem, S. Zikic, and G. E. Faulkner, "Multiple input multiple output systems for optical wireless: Challenges and possibilities," vol. 6304, 2006.

[6] D. V. Plant and A. G. Kirk, "Optical interconnects at the chip and board level: challenges and solutions," Proc. IEEE, vol. 88, no. 6, pp. $806-$ 818, 2000.

[7] E. Bisaillon, D. F. Brosseau, T. Yamamoto, M. Mony, E. Bernier, D. Goodwill, D. V. Plant, and A. G. Kirk, "Free-space optical link with spatial redundancy for misalignment tolerance," IEEE Photonics Technology Letters, vol. 14, no. 2, pp. 242 - 244, 2002.

[8] S. Hranilovic and F. R. Kschischang, "A pixelated MIMO wireless optical communication system," IEEE J. Sel. Topics Quantum Electron. vol. 12, no. 4, pp. 859-874, Jul/Aug 2006.

[9] M. D. A. Mohamed and S. Hranilovic, "Two-dimensional binary halftoned optical intensity channels," IET Communications (formerly, IEE Proceedings Communications), Special Issue on Optical Wireless Communication Systems, vol. 2, no. 1, pp. 11-17, Jan. 2008.

[10] J. Goodman, Introduction to Fourier Optics, 1st ed. McGraw-Hill, June 1968.

[11] D. Bushuev, D. Kedar, and S. Arnon, "Analyzing the performance of a nanosatellite cluster-detector array receiver for laser communication," IEEE/OSA J. Lightw. Technol., vol. 21, no. 2, pp. 447-455, Feb. 2003.

[12] J. M. Kahn and J. R. Barry, "Wireless infrared communications," Proc. IEEE, vol. 85, no. 2, pp. 263-298, Feb. 1997.

[13] Texas Instruments, Digital Light Processing. http://www.dlp.com.

[14] B. L. Shoop, Photonic Analog-to-Digital Conversion. Berlin, Germany: Springer-Verlag, 2001.

[15] R. W. Floyd and L. Steinberg, "An adaptive algorithm for spatial gray scale," in SID Symposium Digest, Society for Information Display, 1975, pp. 36-37.

[16] D. Anastassiou, "Error diffusion coding for A/D conversion," IEEE Trans. Circuits Syst., vol. 36, no. 9, pp. 1175-1186, Sept. 1989.

[17] B. L. Shoop and E. K. Ressler, "An error diffusion neural network for digital image halftoning," in Proc. IEEE Workshop on Neural Networks for Signal Process., 31 Aug.-2 Sept. 1995, pp. 427-436.

[18] S. R. Norsworthy, R. Schreier, and G. C. Temes, Eds., Delta-Sigma Data Converters: Theory, Design, and Simulation. NY, USA: Wiley-IEEE Press, Oct. 1996.

[19] D. A. Johns and K. W. Martin, Analog Integrated Circuit Design, 1st ed. John Wiley \& Sons, Nov. 1996.

[20] NEC Inc. http://www.nec.com/.

[21] Mikrotron GmbH. http://www.mikrotron.de/.

[22] Matrox Imaging. http://www.matrox.com/.

[23] NorPix Inc. http://www.norpix.com/.

[24] National Instruments Inc. http://www.ni.com/. 